\title{
Investigation of Bioactive Properties in a Selected Species from the Leguminosae Plant Family Found in Guyana
}

\author{
Article by Iran Ali \\ Business Administration, Texila American University \\ E-mail:ali_darkice@yahoo.com
}

\begin{abstract}
Plants have always been a vital source of medicine since the dawn of time for man. The pharmacological evaluation of substances from plants is an established method for the identification of lead compounds which can leads to the development of novel and safe medicinal agents. Based on literature the Fabaceae or Leguminosae plant family has medicinal properties which have been proved safe for usage on humans. Species such as Cassia fistula, Saraca asoca, Cassia auriculata and Cassia tora have laxative, treating gynecologic disorders, anti-dysentery and treating skin disease respectively are from the Caesalpinioideae; a sub-family of the Fabaceae or Leguminosae plant family. Most of these botanicals contain heterogenous products in them. These products are bioactive in nature i.e. they are secondary metabolites which aids in the protection and survivability of the plant. These secondary metabolites are compounds such as alkaloids, terpenoids, steroids and polyketides etc. To extract these bioactive compounds from the plant, polar, semi-polar and non-polar solvents are used sequentially for extraction. To help identify if a plant has bioactive properties special bioassays are used which are an inexpensive to carry out on the botanicals. Bioassays offer a special advantage in the standardization and quality control of heterogeneous botanicals products. One such bioassay is the brine shrimp lethality test (BST) a general bioassay. The brine shrimp lethality test (BST) is used to predict the presence, in the plant extracts, cytotoxic activity. This bioassay uses the micro-organisms Artemia salina which is placed in different concentration levels of the plant extracts. The percentage death tells how toxic the plant extract is. The BST is especially sugessted as an inexpensive, simple and rapid means of standardization of bioactivity in heterogeneous botanical products.
\end{abstract}

Keywords: Artemia salina, brine shrimp lethality test, secondary metabolites, cytotoxic activity, medicinal plants, heterogeneous botanical.

\section{Introduction}

Plants are the first source of medicine that was used for both humans and animals. Even now at present many plant species are being studied for the presence of medicinal properties using various bio-assays to check for these properties. Without plants life as we know it would not exist on earth, since plants produce oxygen and food for all living things to survive. Plants are a major group of life forms and include familiar organisms such as trees, herbs, bushes, grasses, vines, ferns, mosses, and green algae. About 350,000 species of plants, defined as seed plants, bryophytes, ferns and fern allies, are estimated to exist currently. As of 2004 , some 287,655 species had been identified, of which 258,650 are flowering and 15,000 bryophytes. The plantae kingdom is divided in to many families some of which has herbal or medicinal properties. Since the dawn of time man have been using plants for many medicinal purposes for sickness. This is because plants contain in them many secondary metabolites that are developed during growth. These secondary metabolites are small molecules like alkaloids, terpenoids, phenols and steroid etc. However then man did not know exactly what was in the plants that made them had healing properties. But in today's technological world and the many bio-assays experimental methods can help in identifying these bioactive compounds in plants. Bioassays offer a special advantage in the standardization and quality control of heterogeneous botanical products. Such products can be "heterogeneous" due to the presence 
of mixtures of bioactive components either from the same or from purposefully mixed botanical sources. One plant family that many studies are being done on or that has been done on is the Fabaceae or Leguminosae.

Investigations of the plants family mentioned above may prove to be helpful to Guyana's development as country, since the countries biodiversity is untouched. Bioactive properties if any to be found in these plant families are of herbal, medicinal and pesticide components can open new ways for the agricultural industry. Since these chemical components are of natural origin i.e. synthesized by plants, they will have almost no harmful effect on the environment. Another advantage of extracting chemical components from the plant species is that they are of abundance in the country; hence production of any useful bioactive properties will be of great benefit to the country's economy. Thus it will be cheap to cultivate any one of these plant family on a large scale.

\section{Objectives}

The experimental research to be carried out on the plant family will enable the Guyanese biodiversity to expand since most of Guyana's plant life is under researched.

- To investigate the bioactive properties in a selected Leguminosae plant family using cytotoxicity bio-assays.

- To prepare crude extracts for the selected bioassay.

- To determine which solvent is most effective in extracting the bioactive properties from the plant species.

\section{Literature review}

Extensive work has been done on the plant family worldwide, but none has apparently been done in Guyana. Also they are used as perfumes. Studies show that these species possesses many useful chemical components with bioactive properties that could be beneficial to the herbal and cosmetics industries (Nor Azah et al. 2001, Zaridah et al. 2003). Many plants synthesize substances that are useful to the maintenance of health in humans and other animals. These include aromatic substances, most of which are phenols or their oxygensubstituted derivatives such as tannins. Many are secondary metabolites, of which at least 12,000 have been isolated - a number estimated to be less than $10 \%$ of the total. In many cases, these substances (particularly the alkaloids) serve as plant defense mechanisms against predation by microorganisms, insects, and herbivores. One such plant family is the Fabaceae or Leguminosae are a large and economically important family of flowering plants, which is commonly known as the legume family, pea family, bean family or pulse family. It is the third largest family of flowering plants (after Orchidaceae and Asteraceae) with 730 genera and over 19,400 species, according to the Royal Botanical Gardens. The Fabaceae comprise three subfamilies (with distribution and some representative species):

- Mimosoideae: 80 genera and 3,200 species. Mostly tropical and warm temperate Asia and America. Mimosa, Acacia.

- Caesalpinioideae: 170 genera and 2,000 species, cosmopolitan. Senna, Cassia.

- Faboideae: 470 genera and 14,000 species, cosmopolitan. Astragalus, Lupinus.

The Fabaceae plant family contains species such as the Dolichos biflorus, Pongamia pinnata, Mucuna pruriens, Glycyrrhiza glabra and Teramnus labialis are used in diuretic, anti-viral, anti-parkinsonism, anti-inflammatory and paralysis respectively ( International Journal of Applied Science and Engineering 2005. 3,2: 125-134).

Below are a few bioactive compounds found in plants: 


\section{Ephedrine}

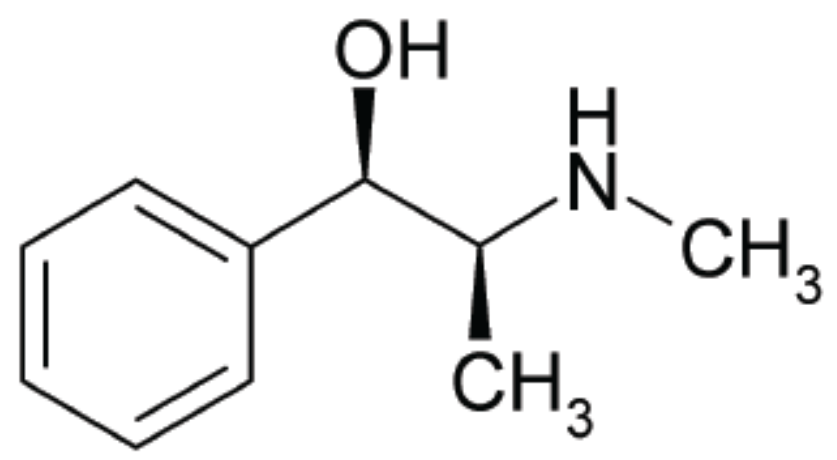

\section{Morphine}

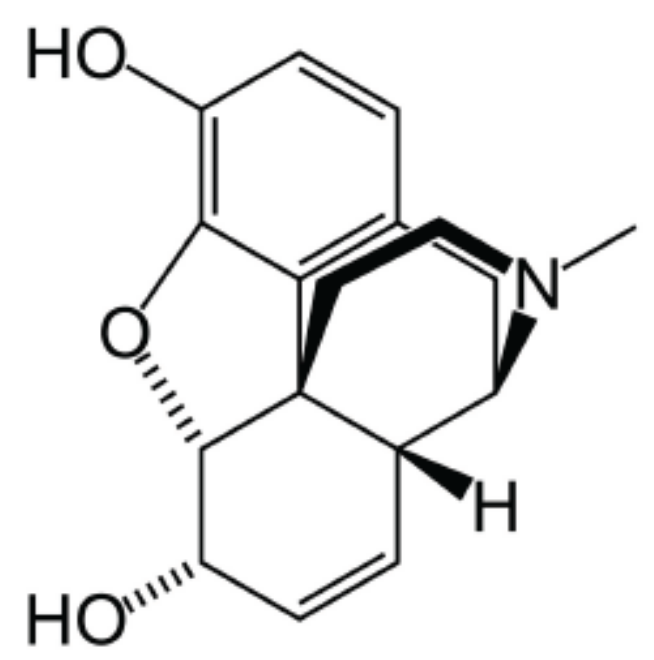

\section{Methodology}

Collection and extraction: the plant species was collected at the Annandale Primary School form one tree and identified at the University of Guyana Biodiversity center. The bark, stems, leaves and pods were collected and put to air dry for 2-4 weeks. After drying the plant parts that were collected was cut and crushed into small pieces. Then 200 grams of each plant parts were weighed and placed in jars. Each jar was then filled with Dichloromethane to extract the non-polar constituents first. The plant parts were soaked for 2-3 days and then the solvent was decanted and a second soaking was done for another 2-3 days. This same procedure was carried out for the methanol solvent. The extracts were then placed in a rotation evaporation to take off the solvents. The crude extracts were then weighed and kept for the bio-assay.

\section{Preparation of brine shrimp larvae}

The plant extracts were then tested for cytotoxcity using brine shrimp. The sea water was prepared according to directions on box (38g sea salt per litre of water). The sea water was placed in small tank, and the brine shrimp larva was added to one side of the divided tank and was covered. A yeast solution was then added for food for the larvae. A lamp was placed on the other side to attract the hatched larvae. A period of $48 \mathrm{hr}$ was allowed for the shrimp to hatched and mature as nauplii.

\section{Brine shrimp microwell cytoxicity assay}

Each plant extract solutions were tested at concentrations of 1000,100 , and $10 \mathrm{mcg} / \mathrm{ml}$ (ppm). Three vials were prepared at each concentration for a total of nine vials. $20 \mathrm{mg}$ of extract was weighed out and dissolved in $2 \mathrm{ml}$ of the solvent and then $0.5 \mathrm{ml}$ was removed 
DOI: $10.21522 /$ TJJAR.2014.04.02.Art017

ISSN: $2520-3088$

using a $1 \mathrm{ml}$ syringe 3 times for the $1000 \mathrm{ppm}$ concentration. $0.2 \mathrm{ml}$ was removed and $1.8 \mathrm{ml}$ solvent was added to make up the $100 \mathrm{ppm}$ concentration. The same procedure was done for the remaining concentration. The vials were left in open for the solvent to evaporate. Then 5 $\mathrm{ml}$ of the brine water was added to the vials, along with 10 brine shrimp and 1-2 drops of yeast solution. The vials were then placed in light for 24 hours. After 24 hours later the number of survivors were counted and recorded. The SPSS program was used for data analysis.

\section{Results}

Table 1. Showing number of survivors in control

\begin{tabular}{|l|l|l|l|}
\hline \multirow{2}{*}{} & \multicolumn{3}{|l|}{ VIALS } \\
\cline { 2 - 4 } & 1 & 2 & 3 \\
\hline SOLVENTS & & & \\
\hline DCM & 0 & 0 & 0 \\
\hline $\mathrm{MeOH}$ & 1 & 0 & 0 \\
\hline
\end{tabular}

Table 2. Showing number of survivors in plant extracts for DCM

\begin{tabular}{|l|l|l|l|}
\hline & \multicolumn{3}{l}{ VIALS } \\
\hline Plant extracts/ppm & 1 & 2 & 3 \\
\hline DCM Bark/1000 & 0 & 0 & 0 \\
\hline 100 & 0 & 0 & 0 \\
\hline 10 & 1 & 2 & 2 \\
\hline DCM Leaves/1000 & 2 & 0 & 0 \\
\hline 100 & 4 & 0 & 0 \\
\hline 10 & 3 & 0 & 0 \\
\hline DCM Stem/1000 & 0 & 0 & 0 \\
\hline 100 & 0 & 1 & 1 \\
\hline 10 & 0 & 2 & 0 \\
\hline DCM Pod/1000 & 0 & 0 & 0 \\
\hline 100 & 0 & 1 & 0 \\
\hline 10 & 5 & 3 & 0 \\
\hline
\end{tabular}

Table 3. Showing number of survivors in plant extracts for methanol

\begin{tabular}{|l|l|l|l|}
\hline $\mathrm{MeOH}$ Bark/1000 & 0 & 0 & 0 \\
\hline 100 & 0 & 0 & 0 \\
\hline 10 & 0 & 0 & 0 \\
\hline $\mathrm{MeOH}$ Leaves/1000 & 0 & 0 & 0 \\
\hline 100 & 0 & 0 & 0 \\
\hline 10 & 0 & 1 & 0 \\
\hline $\mathrm{MeOH}$ Stem/1000 & 0 & 0 & 0 \\
\hline 100 & 0 & 0 & 0 \\
\hline 10 & 1 & 0 & 0 \\
\hline $\mathrm{MeOH}$ Pod/1000 & 0 & 0 & 0 \\
\hline 100 & 0 & 0 & 0 \\
\hline 10 & 1 & 0 & 0 \\
\hline
\end{tabular}

Percentage death $=\underline{\text { number died in test- number died in control }}$

Percentage death $=\underline{10-10 \times 100}$

$$
\text { Number exposed }
$$

$=100 \%$ 


\section{Discussion}

The SPSS program was used to analyze the data but since the percentage death was high it could not be computed using the program. There was $100 \%$ death of the brine shrimp in the controls using the solvents; hence the solvents are toxic to the organisms. From the table it can be seen that at concentrations level above 1000 and 100 ppm there was $100 \%$ death of the brine shrimp for both solvents, this can be linked to the fact that this plant family contain bioactive properties (secondary metabolites) such as alkaloids, aromatic compounds and phenolic compounds which is according to literature. For the methanol extracts only 1 brine shrimp survived at concentration $10 \mathrm{ppm}$ for the pod, stem and leaves. This may due to the methanol was used to extract the polar constituents which are more toxic to the larvae, compared to the DCM extracts at concentration $10 \mathrm{ppm}$ for all the plant parts there were more survivors thus the non-polar constituents are less toxic at concentrations below $10 \mathrm{ppm}$. However for both solvents less than half of the larvae survived.

\section{Conclusions}

From the bio-assay carried out using the brine shrimp lethality, it is conclusive that the concentrations levels above $100 \mathrm{ppm}$ are very toxic to the larvae for both the solvents used. Thus concentrations of $10 \mathrm{ppm}$ and below are less toxic to the larvae for the DCM extracts and for the methanol extracts it is still toxic to the larvae. The methanol proved to be better solvent for extraction used on the plant. It is also conclusive that this plant family contains bioactive properties.

\section{Recommendations}

Future work can be carried out on the same plant species using higher mass for extractions and also using different solvents such as ethanol and hexane. Also lower concentrations level of less than $10 \mathrm{ppm}$ can be used in the brine shrimp lethality.

\section{References}

[1]. Antimicrobial activity and brine shrimp toxicity of extracts Terminalia bromnii roots and stem; http://www.pubmedcentral.nih.gov/articlerender.fcgi?artid=1851717.

[2]. Alluri V. Krishnaraju, Tayi V. N. Rao, Dodda Sundararaju, Mulabagal Vanisree, Hsin-Sheng Tsay and Gottumukkala V. Subbaraju; Assessment of Bioactivity of Indian Medicinal Plants Using Brine Shrimp Lethality Assay; International Journal of Applied Science and Engineering 2005. 3, 2: 125-134.

[3]. Baby brine shrimp; http://www.bettatalk.com/hatching_brine_shrimp.htm.

[4]. Caesalpinioideae; http://en.wikipedia.org/wiki/Caesalpinioideae.

[5]. Dichloromethane; http://en.wikipedia.org/wiki/Dichloromethane.

[6]. Fabaceae; http://en.wikipedia.org/wiki/Fabaceae.

[7]. Jerry L. McLaughlin, PhD and Lingling L. Rogers, Ms; The use of biological assays to evaluate botanicals; Drug Information Journal, Vol. 32, pp. 513-524, 1998.

[8]. Methanol; http://en.wikipedia.org/wiki/Methanol.

[9]. Natural product drug discovery; http://en.wikipedia.org/wiki/Natural_product_drug_discovery.

[10]. Q.A. Nguyen $e t$ al; A new Phenylpropaniod Ester; August $2^{\text {nd }}, 2002$.

[11]. Secondary metabolites; http://en.wikipedia.org/wiki/Secondary_metabolite.

[12]. Screening of Argentine Medicinal Plants using the Brine Shrimp Micro well Cytotoxicity Assay; International Journal of Pharmacognosy; 1996; Vol. 34, No. 4, pp. 249-254.

[13]. The use of spices and medicinal as Bioactive for Protectants;

http://www.fao.org/docrep/x2230e/x2230e09.htm. 